\title{
GENETIC ALGORITHM PARAMETERS OPTIMIZATION FOR ELECTROCHEMICAL MACHINING USING RESPONSE SURFACE METHODOLOGY
}

\author{
Vikas Kumar ${ }^{1} \&$ Dr. Ashwani Kumar Dhingra ${ }^{2}$
}

Abstract-In this paper, an attempt has been made for the judgment of the optimum parameters of GA for the optimization of Material Removal Rate (MRR) and the Surface Roughness (SR) simultaneously in Electro Chemical Machining (ECM) using Response Surface Methodology (RSM). Performance of GA can be achieved by optimization of its various parameters, i.e. creation function, crossover fraction, mutation function, population size etc. Fitness functions has been developed in GA for maximizing the MRR and minimization of SR. Combinational parameters of GA have been obtained by 5 levels central composite design approach of Response Surface Methodology (RSM) The optimization of the process parameter is primarily based on the working parameter used by the operator which is not possible that every time operator work on the optimum parameters. Due to this aspect GA optimization tool is required for effective utilization of the process. The main aim of this paper is to get the optimum GA parameters for maximizing the Material removal rate (MRR) and minimizing surface roughness (SR) for the Electro Chemical Machining (ECM) simultaneously.

Keywords-Electro Chemical Machining(ECM), Genetic algorithm, Regression model, Response surface methodology, Material Removal Rate(MRR), Surface Roughness (SR).

\section{INTRODUCTION}

Electrochemical machining (ECM) is one of the most broadly used nontraditional machining processes, which is used to machine extremely hard materials. ECM is a method of removing metal by electrochemical process.ECM has characterized as reverse electroplating and working on the principles of Faraday (1833).Faraday's law states that the mass of a metal distorted by the electrode is proportional to the quantity of electrical charges transferred to that electrode. A potential difference is maintained between electrodes as a result the ions existing in the electrolyte transfer toward the electrode. The desired amount of metal is removed because of ions movement towards the tool. In ECM the removal of metal is controlled by the anodic dissolution in the electrolyte. In ECM work piece act as anode and tool act as cathode. The electrolyte should be placed narrow in the gap of about $0.5 \mathrm{~mm}$. The anode and cathode should be placed deep into the electrolyte. A genetic algorithm is used for optimizing the process parameters of the ECM machine. A genetic algorithm is a population based approach which is very effective in solving very hard problems like optimization of process parameters of various machines .GA is global, robust and anyone can apply it in such a situation where a little or a priori knowledge about the process and how to control it . Genetic algorithm normally starts with a preliminary population of chromosomes, which are randomly produced and based on several algorithms, other policies and Socratics.

Bhattacharyya et al (2005) highlighted various electrochemical micro-machining parameters like machining voltage, frequency, pulse period and electrolyte concentration and their influences on the Material Removal Rate (MRR), surface finish and accuracy in infinitesimal field. His study shows that $3 \mathrm{~V}$ machining voltage, $20 \mathrm{~g} / \mathrm{l}$ electrolyte concentration and $55 \mathrm{~Hz}$ frequency are optimal values of Electro chemical machining that can augment the accurateness with the uppermost possible amount of material removed (MRR). N. K. Jain et al (2007) concentrates his work on the optimization of tool feed rate, applied voltage, and electrolyte flow velocity with the purpose to minimize the geometrical inaccuracy subjected to choking, passivity constraint and temperatures. Via single objective real-coded genetic algorithms for obtaining the optimization process parameter of ECM machine after those optimum results were verified graphically and theoretically. His work shows that the optimum process parameters can extensively progress the process recital and progression economics by dropping operating, maintenance cost and tooling cost and manufacturing components of higher precision also try to extend

\footnotetext{
${ }^{1}$ Corresponding author Department of Mechanical Engineering, University Institute of Engineering and Technology, Maharishi Dayanand University, Rohtak, Haryana (124001) India

${ }^{2}$ Department of Mechanical Engineering, University Institute of Engineering and Technology, Maharishi Dayanand University, Rohtak, Haryana (124001) India
} 
tool life, machining cost and machining time by using suitable evolutionary algorithms. Dilip Datta et al (2010) applied a multi-objective GA to electrochemical machining for obtaining the optimum diverse process parameters by taking a tentative dataset for sculpting the problem through regression equation after that applied the genetic algorithm to linear model and an exponential model for obtaining the maximum MRR and minimizing the surface roughness (SR) simultaneously. Chinnamuthu Senthilkumar et al (2012) developed a second order polynomial arithmetical models for linking the interactive and higher-order of diverse machining parameters in the machining standard I,E. MRR and SR by using response surface methodology. They test models by ANOVA test. After that Non-dominated Sorting Genetic Algorithm-II (NSGA-II) was applied to get the optimized process parameter for exploit material removal rate and diminish surface roughness. Optimized value attained through NSGA-II, is $5.020 \mu \mathrm{m}$ and the consequent MRR is $0.831 \mathrm{gm} / \mathrm{min}$ and the pertinent constraints were , electrolyte flow rate, electrolyte concentration applied voltage and tool feed rate are 5 lit/min, $16 \mathrm{gm} / \mathrm{lit}$, $15 \mathrm{volts}$ and 1.0 $\mathrm{mm} / \mathrm{min}$ correspondingly. Biswesh R. Acharya et al (2013) used a central composite design for response surface methodology to study the effect of 4 different constraints (current, flow rate of electrolyte, voltage , and inter-electro gap) on Material Removal rate (MRR) and Surface roughness (SR) of the hardened steel material on ECM machine and also they develop empirical equation for MRR and SR and tested for statistical study on four parameters of ECM machine. After that they use a non-dominated sorted genetic algorithm to find out the optimal process parameter that simultaneously maximizes MRR and minimize SR and the validation of optimum result can be done by doing electrochemical machining for the corresponding input parameters. A Giribabu et al (2014) used L27 orthogonal array and genetic algorithms for optimizing the machining parameters for electrochemical machining of Al/B4C composites in that they considered four main parameters applied voltage (electrical constraint),tool feed rate(electrode constraint), electrolyte concentration (electrolyte constraint) and reinforcement content (work piece property) as input machining constraints and also developed a regression models for Material removal Rate, Surface roughness and Radial Over Cut and then use that regression equation in genetic algorithms for getting the optimum machinating parameters to maximize Material removal rate(MRR), minimize Surface roughness(SR) and minimize Radial Over Cut (ROC) simultaneously. M. Sankar et al (2014) used response surface methodology (RSM) relevance to optimize the machining constraints with multiple response method in electrochemical machining (ECM) of a 7075Al/B4C metal matrix compound and after obtaining the optimum parameter the result was compared with ANOVA. The result what they obtain through after applied the response surface methodology (RSM) demonstrate that feed rate and voltage are the most considerable constraint which affect multiple machining responses concurrently and the responses in ECM can be improved successfully through this loom. The optimum results given by the experiment are as follows $8 \mathrm{~V}$ voltage and $0.3 \mathrm{~mm} / \mathrm{min}$ feed rate and 217A current are most appropriate and momentous to achieve utmost surface finish with NaNO3 electrolyte solution. V. Sathiyamoorthy et al (2015) attempted to diminish the formation of spikes in the work piece of high carbon and high chromium die tool steel using nano particles of copper floating in plain NaNO3 electrolyte. They used Design expert 7.0 software to developed the mathematical models for getting the response like MRR and SR and after that multi-objective genetic algorithm in MATLAB was used for the best probable combination for accomplishing the maximum MRR and minimum SR. After comparing the result from design expert 7.0 to the optimum results obtained by using multi-objective genetic algorithm (MOGA) the conclusion come that the distinction from the predicted performance is less than $4 \%$ which verify the composite popularity of the developed models. Abhishek Tiwari et al (2015) done the optimization of ECM for EN 19 tool steel by using Non- dominated sorting Genetic Algorithm-II (NSGA-II) for maximizing the MRR and minimizing the SR. They prepared a mathematical model for correlating the input and output parameters and ANOVA test was done on that to show the concentrate- ion and feed rate was most dominating criteria for MRR and SR respectively. They also represent the optimal machine parameter for ECM machine through Non-dominated Sorting Genetic Algorithm-II (NSGA-II).

\subsection{Problem statement}

ECM is widely used in various industries. Quality and productivity of a product are highly considered. There are diverse parameters which affect the quality of the product and productivity. The MRR and SR are the prime response factors for higher productivity and good quality respectively. Improper combinational process parameters leads in productivity reduction and for the poor quality. So, we need to optimize the process parameters for maximizing the MRR and minimize the SR . Previously, research work has been done on optimizing the machine parameter for maximizing the MRR and minimizing the SR by using various mathematical and computational models. The ranges of the selected input parameters and regression equation used in this study are taken from (Rao and Padmanabhan, 2013). With the help of these regression equations the fitness function is created in GA for getting the values of MRR and SR.

$\mathrm{MRR}=-0.166+0.0272 \mathrm{X} 1+0.424 \mathrm{X} 2+0.00776 \mathrm{X} 3-0.0284 \mathrm{X} 4$

$\mathrm{SR}=5.04+0.0153 \mathrm{X} 1-0.648 \mathrm{X} 2-0.0292 \mathrm{X} 3+0.0551 \mathrm{X} 4$

And there limits are as follow

Applied voltage

Feed rate

Electrolyte concentration

Percentage of reinforcement
$12 \square X 1 \square 20$

$0.2 \square X 2 \square 1$

$10 \square X 3 \square 30$

$2.5 \square X 4 \square 7.5$ 


\section{METHODOLOGY}

In GA every generation of the population goes throughout various progressions like encoding, Fitness function valuation selection, crossover and transformation. Genetic Algorithms work on the principles of natural genetics to evolve solutions of the problems .A Script fitness function is created in GA according to our requirement. When we execute this fitness function each set of the population represent a solution of the problem. Crossover \& Mutation are used for producing new offsprings which are known as Reproduction operators. In this two Mutation function (Uniform and Adaptive feasible) and three crossover functions (Scattered, Single point and two points) are used for optimization. GA represents a novel programming pattern that attempts to copy the course of natural evolution, to resolve computing and optimization problems of computers. In GA, a population of chromosomes, which are generally twine of bits, is arbitrarily selected. This population further altered into a new population by a variety of natural selection based on the utilize of the operators encouraged by the natural genetic operatives.

The natural selection is fully based on the output of the function which is called fitness function. Only the best fit chromosomes are survived and permissible to further reproduce the new offsprings or we can say these are used in the next population. After exchanging the properties of two parents with each other and as a result of which two children created or making arbitrary changes to single parent by mutation. By replacing the present population with the children to structure the new generation. The algorithm end when the generation of a new population had completed.

The complete work is separated into two parts: first part is finds the maximum MRR and minimum SR simultaneously uses GA and the second part optimize the parameters of GA using Design expert. In this approx. 156 experiments were obtained by using 5 level central composite design approaches for Response surface methodology in design expert software are shown in Table 1.

Table1: Various Parameters Combination Of Ga By Using Design Expert

\begin{tabular}{|c|c|c|c|c|c|c|c|c|}
\hline Std & & Factor 1 & \multicolumn{2}{|r|}{ Factor2 } & \multirow{2}{*}{\multicolumn{2}{|c|}{ Factor3 }} & Factor4 \\
\hline Factor5 & \multicolumn{2}{|c|}{ Response1 } & Response2 & \multirow{2}{*}{\multicolumn{2}{|c|}{ B:Crossover }} & & & \\
\hline & & & \multirow{2}{*}{$\begin{array}{l}\text { A:Population } \\
\text { SR }\end{array}$} & & & \multicolumn{2}{|c|}{ C:Creation } & D:Mutation \\
\hline E:Crossover & \multicolumn{2}{|c|}{ MRR } & & & size & fraction & function & function \\
\hline \multicolumn{9}{|l|}{ function } \\
\hline $\begin{array}{l}16 \\
3.889\end{array}$ & 1 & 20 & 0.9 & \multicolumn{2}{|c|}{ Feasible population } & Uniform & Scattered & 0.68323 \\
\hline 63 & 2 & 45 & 0.7 & Uniform & Uniform & Single point & 0.7174 & 3.894 \\
\hline 2 & 3 & 70 & 0.4 & Uniform & Uniform & Scattered & 0.7242 & 3.8588 \\
\hline 106 & 4 & 70 & 0.4 & Uniform & Uniform & Two point & 0.7328 & 3.8708 \\
\hline 55 & 5 & 20 & 0.9 & Uniform & Uniform & Single point & 0.7222 & 3.9126 \\
\hline 9 & 6 & 45 & 0.7 & Uniform & Uniform & Scattered & 0.6938 & 3.892 \\
\hline 116 & 7 & 45 & 0.7 & Uniform & Uniform & Two point & 0.7348 & 3.8688 \\
\hline 95 & 8 & 70 & 0.9 & \multicolumn{2}{|c|}{ Feasible population } & \multicolumn{2}{|c|}{ Adaptive feasible } & Single point \\
\hline 0.743 & 3.8448 & & & & & & & \\
\hline 13 & 9 & 45 & 0.7 & Uniform & Uniform & Scattered & 0.746 & 3.8282 \\
\hline 86 & 10 & 45 & 1.0 & Uniform & \multicolumn{2}{|c|}{ Adaptive feasible } & Single point & 0.7288 \\
\hline 3.882 & & & & & & & & \\
\hline 77 & 11 & 45 & 0.7 & \multirow{2}{*}{\multicolumn{2}{|c|}{ Feasible population }} & Uniform & Single point & 0.7318 \\
\hline 3.7984 & & & & & & & & \\
\hline $\begin{array}{l}35 \\
3.8487\end{array}$ & 12 & 45 & 0.7 & Uniform & \multicolumn{2}{|c|}{ Adaptive feasible } & Scattered & 0.7424 \\
\hline 1 & 13 & 20 & 0.4 & Uniform & Uniform & Scattered & 0.7122 & 3.9632 \\
\hline 145 & 14 & 70 & 0.4 & Feasible $\mathrm{p}$ & pulation & Adaptive fea & asible & Two point \\
\hline 0.746 & 3.8405 & & & & & & & \\
\hline $\begin{array}{l}30 \\
3.8408\end{array}$ & 15 & 70 & 0.9 & Uniform & Adaptive & asible & Scattered & 0.7458 \\
\hline $\begin{array}{l}141 \\
3.8386\end{array}$ & 16 & 45 & 0.7 & Uniform & Adaptive & asible & Two point & 0.747 \\
\hline $\begin{array}{l}132 \\
3.8474\end{array}$ & 17 & 70 & 0.4 & Uniform & Adaptive & asible & Two point & 0.7446 \\
\hline $\begin{array}{l}20 \\
3.875\end{array}$ & 18 & 45 & 0.3 & Feasible $\mathrm{p}$ & pulation & Uniform & Scattered & 0.7366 \\
\hline 53 & 19 & 20 & 0.4 & Uniform & Uniform & Single point & 0.73 & 3.94 \\
\hline
\end{tabular}


Genetic Algorithm Parameters Optimization For Electrochemical Machining Using Response Surface

Methodology

\begin{tabular}{|c|c|c|c|c|c|c|c|c|}
\hline \multirow{4}{*}{$\begin{array}{l}150 \\
0.746 \\
71 \\
3.8637\end{array}$} & \multirow{2}{*}{$\begin{array}{l}20 \\
3.838\end{array}$} & \multirow[t]{2}{*}{45} & \multirow{2}{*}{0.3} & \multirow{2}{*}{\multicolumn{2}{|c|}{ Feasible population }} & \multirow{2}{*}{\multicolumn{2}{|c|}{ Adaptive feasible }} & \multirow{2}{*}{ Two point } \\
\hline & & & & & & & & \\
\hline & 21 & 80 & 0.7 & Feasible & pulation & Uniform & Single point & 0.7284 \\
\hline & & & & & & & & \\
\hline $\begin{array}{l}140 \\
3.8394\end{array}$ & 22 & 45 & 0.7 & Uniform & \multicolumn{2}{|c|}{ Adaptive feasible } & Two point & 0.7484 \\
\hline $\begin{array}{l}130 \\
3.895\end{array}$ & 23 & 45 & 0.7 & \multicolumn{2}{|c|}{ Feasible population } & Uniform & Two point & 0.7542 \\
\hline 112 & 24 & 45 & 1.0 & Uniform & Uniform & Two point & 0.7352 & 3.8824 \\
\hline $\begin{array}{l}135 \\
3.9342\end{array}$ & 25 & 10 & 0.7 & Uniform & \multicolumn{2}{|c|}{ Adaptive feasible } & Two point & 0.741 \\
\hline $\begin{array}{l}69 \\
3.868\end{array}$ & 26 & 70 & 0.9 & \multicolumn{2}{|c|}{ Feasible population } & Uniform & Single point & 0.7308 \\
\hline $\begin{array}{l}143 \\
3.838\end{array}$ & 27 & 45 & 0.7 & Uniform & \multicolumn{2}{|c|}{ Adaptive feasible } & Two point & 0.748 \\
\hline 111 & 28 & 45 & 0.3 & Uniform & Uniform & Two point & 0.7356 & 3.8514 \\
\hline 7 & 29 & 45 & 0.3 & Uniform & Uniform & Scattered & 0.71662 & 3.8642 \\
\hline $\begin{array}{l}120 \\
3.9634\end{array}$ & 30 & 20 & 0.9 & \multicolumn{2}{|c|}{ Feasible population } & Uniform & Two point & 0.6884 \\
\hline $\begin{array}{l}37 \\
3.838\end{array}$ & 31 & 45 & 0.7 & Uniform & \multicolumn{2}{|c|}{ Adaptive feasible } & Scattered & 0.746 \\
\hline 23 & 32 & 45 & 0.7 & \multicolumn{2}{|c|}{ Feasible population } & Uniform & Scattered & 0.7342 \\
\hline 61 & 33 & 45 & 0.7 & Uniform & Uniform & Single point & 0.7456 & 3.894 \\
\hline 5 & 34 & 10 & 0.7 & Uniform & Uniform & Scattered & 0.67392 & 4.0304 \\
\hline 113 & 35 & 45 & 0.7 & Uniform & Uniform & Two point & 0.7158 & 3.894 \\
\hline $\begin{array}{l}139 \\
3.8394\end{array}$ & 36 & 45 & 0.7 & Uniform & \multicolumn{2}{|c|}{ Adaptive feasible } & Two point & 0.7476 \\
\hline $\begin{array}{l}134 \\
3.8412\end{array}$ & 37 & 70 & 0.9 & Uniform & \multicolumn{2}{|c|}{ Adaptive feasible } & Two point & 0.7438 \\
\hline $\begin{array}{l}94 \\
0.7428\end{array}$ & $\begin{array}{l}38 \\
3.8478\end{array}$ & 20 & 0.9 & \multicolumn{2}{|c|}{ Feasible population } & \multicolumn{2}{|c|}{ Adaptive feasible } & Single point \\
\hline $\begin{array}{l}19 \\
3.864\end{array}$ & 39 & 80 & 0.7 & \multicolumn{2}{|c|}{ Feasible population } & Uniform & Scattered & 0.7326 \\
\hline 65 & 40 & 45 & 0.7 & Uniform & Uniform & Single point & 0.7352 & 3.8672 \\
\hline $\begin{array}{l}40 \\
0.746\end{array}$ & $\begin{array}{l}41 \\
3.9245\end{array}$ & 20 & 0.4 & \multicolumn{2}{|c|}{ Feasible population } & \multicolumn{2}{|c|}{ Adaptive feasible } & Scattered \\
\hline $\begin{array}{l}102 \\
0.7404\end{array}$ & $\begin{array}{l}42 \\
3.8496\end{array}$ & 45 & 0.7 & \multicolumn{2}{|c|}{ Feasible population } & \multicolumn{2}{|c|}{ Adaptive feasible } & Single point \\
\hline $\begin{array}{l}41 \\
0.746\end{array}$ & $\begin{array}{l}43 \\
3.837\end{array}$ & 70 & 0.4 & \multicolumn{2}{|c|}{ Feasible population } & \multicolumn{2}{|c|}{ Adaptive feasible } & Scattered \\
\hline $\begin{array}{l}22 \\
3.91\end{array}$ & 44 & 45 & 0.7 & Feasible & pulation & Uniform & Scattered & 0.7358 \\
\hline $\begin{array}{l}154 \\
0.7458\end{array}$ & $\begin{array}{l}45 \\
3.84\end{array}$ & 45 & 0.7 & Feasible & pulation & Adaptive fea & sible & Two point \\
\hline $\begin{array}{l}122 \\
3.9522\end{array}$ & 46 & 10 & 0.7 & Feasible & pulation & Uniform & Two point & 0.6798 \\
\hline $\begin{array}{l}131 \\
3.9536\end{array}$ & 47 & 20 & 0.4 & Uniform & Adaptive & asible & Two point & 0.7423 \\
\hline $\begin{array}{l}78 \\
3.8318\end{array}$ & 48 & 45 & 0.7 & Feasible & pulation & Uniform & Single point & 0.7304 \\
\hline $\begin{array}{l}96 \\
0.7034\end{array}$ & $\begin{array}{l}49 \\
4.0048\end{array}$ & 10 & 0.7 & Feasible & pulation & Adaptive fea & sible & Single point \\
\hline $\begin{array}{l}127 \\
3.8592\end{array}$ & 50 & 45 & 0.7 & Feasible & pulation & Uniform & Two point & 0.7368 \\
\hline 64 & 51 & 45 & 0.7 & Uniform & Uniform & Single point & 0.7356 & 3.861 \\
\hline 97 & 52 & 80 & 0.7 & Feasible & pulation & Adaptive fea & sible & Single point \\
\hline 0.7446 & 3.8385 & & & & & & & \\
\hline 149 & 53 & 80 & 0.7 & Feasible & pulation & Adaptive fea & sible & Two point \\
\hline
\end{tabular}




\begin{tabular}{|c|c|c|c|c|c|c|c|c|}
\hline 0.746 & 3.837 & & & & \multirow{2}{*}{\multicolumn{2}{|c|}{ Adaptive feasible }} & & \\
\hline $\begin{array}{l}83 \\
3.9242\end{array}$ & 54 & 10 & 0.7 & Uniform & & & Single point & 0.7204 \\
\hline $\begin{array}{l}89 \\
3.8384\end{array}$ & 55 & 45 & 0.7 & Uniform & \multicolumn{2}{|c|}{ Adaptive feasible } & Single point & 0.7464 \\
\hline 151 & 56 & 45 & 1.0 & Feasible & ulation & \multirow{2}{*}{\multicolumn{2}{|c|}{ Adaptive feasible }} & Two point \\
\hline 0.723 & 3.8856 & & & & & & & \\
\hline $\begin{array}{l}87 \\
3.837\end{array}$ & 57 & 45 & 0.7 & Uniform & \multicolumn{2}{|c|}{ Adaptive feasible } & Single point & 0.746 \\
\hline 59 & 58 & 45 & 0.3 & Uniform & Uniform & \multirow{2}{*}{\multicolumn{2}{|c|}{$\begin{array}{l}\text { Single point } 0.7352 \\
\text { Adaptive feasible }\end{array}$}} & 3.8608 \\
\hline $\begin{array}{l}155 \\
0.74836\end{array}$ & $\begin{array}{l}59 \\
3.8424\end{array}$ & 45 & 0.7 & Feasible & ulation & & & Two point \\
\hline $\begin{array}{l}124 \\
3.9002\end{array}$ & 60 & 45 & 0.3 & Feasible & ulation & Uniform & Two point & 0.7334 \\
\hline $\begin{array}{l}17 \\
3.8986\end{array}$ & 61 & 70 & 0.9 & Feasible & ulation & Uniform & Scattered & 0.7106 \\
\hline $\begin{array}{l}68 \\
4.0256\end{array}$ & 62 & 20 & 0.9 & Feasible & ulation & Uniform & Single point & 0.7175 \\
\hline $\begin{array}{l}25 \\
3.8922\end{array}$ & 63 & 45 & 0.7 & Feasible & ulation & Uniform & Scattered & 0.7162 \\
\hline $\begin{array}{l}104 \\
0.746\end{array}$ & $\begin{array}{l}64 \\
3.8378\end{array}$ & 45 & 0.7 & Feasible & ulation & Adaptive & sible & Single point \\
\hline $\begin{array}{l}82 \\
3.846\end{array}$ & 65 & 70 & 0.9 & Uniform & \multicolumn{2}{|c|}{ Adaptive feasible } & Single point & 0.743 \\
\hline $\begin{array}{l}138 \\
3.8794\end{array}$ & 66 & 45 & 1.0 & Uniform & \multicolumn{2}{|c|}{ Adaptive feasible } & Two point & 0.7254 \\
\hline 100 & 67 & 45 & 0.7 & Feasible & ulation & \multirow{2}{*}{\multicolumn{2}{|c|}{ Adaptive feasible }} & Single point \\
\hline 0.746 & 3.828 & & & & & & & \\
\hline 107 & 68 & 20 & 0.9 & Uniform & Uniform & \multirow{2}{*}{\multicolumn{2}{|c|}{$\begin{array}{l}\text { Two point } 0 . / 232 \\
\text { Adaptive feasible }\end{array}$}} & 3.9248 \\
\hline 156 & 69 & 45 & 0.7 & Feasible & ulation & & & Two point \\
\hline 0.7447 & 3.8466 & & & & & & & \\
\hline 4 & 70 & 70 & 0.9 & Uniform & Uniform & Scattered & 0.7144 & 3.8382 \\
\hline $\begin{array}{l}91 \\
3.838\end{array}$ & 71 & 45 & 0.7 & Uniform & \multicolumn{2}{|c|}{ Adaptive feasible } & Single point & 0.746 \\
\hline $\begin{array}{l}52 \\
0.7458\end{array}$ & $\begin{array}{l}72 \\
3.837\end{array}$ & 45 & 0.7 & Feasible & ulation & \multicolumn{2}{|c|}{ Adaptive feasible } & Scattered \\
\hline $\begin{array}{l}98 \\
0.745\end{array}$ & $\begin{array}{l}73 \\
3.84\end{array}$ & 45 & 0.3 & Feasible & ulation & \multicolumn{2}{|c|}{ Adaptive feasible } & Single point \\
\hline $\begin{array}{l}128 \\
3.8824\end{array}$ & 74 & 45 & 0.7 & Feasible & ulation & \multicolumn{2}{|c|}{ 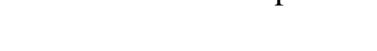 } & 0.7306 \\
\hline $\begin{array}{l}42 \\
0.7452\end{array}$ & $\begin{array}{l}75 \\
3.8392\end{array}$ & 20 & 0.9 & Feasible & ulation & \multicolumn{2}{|c|}{ Adaptive feasible } & Scattered \\
\hline $\begin{array}{l}75 \\
3.8796\end{array}$ & 76 & 45 & 0.7 & Feasible & ulation & Uniform & Single point & 0.7272 \\
\hline $\begin{array}{l}14 \\
4.0256\end{array}$ & 77 & 20 & 0.4 & Feasible & ulation & Uniform & Scattered & 0.7094 \\
\hline $\begin{array}{l}125 \\
3.8854\end{array}$ & 78 & 45 & 1.0 & Feasible & ulation & Uniform & Two point & 0.7268 \\
\hline $\begin{array}{l}85 \\
3.8532\end{array}$ & 79 & 45 & 0.3 & Uniform & \multicolumn{2}{|c|}{ Adaptive feasible } & Single point & 0.7414 \\
\hline $\begin{array}{l}18 \\
4.076\end{array}$ & 80 & 10 & 0.7 & Feasible & ulation & Uniform & Scattered & 0.6748 \\
\hline $\begin{array}{l}92 \\
0.7338\end{array}$ & $\begin{array}{l}81 \\
3.8906\end{array}$ & 20 & 0.4 & Feasible & ulation & Adaptive & ssible & Single point \\
\hline $\begin{array}{l}70 \\
3.9662\end{array}$ & 82 & 10 & 0.7 & Feasible & ulation & Uniform & Single point & 0.6902 \\
\hline 117 & 83 & 45 & 0.7 & Uniform & Uniform & Two poin & 0.706 & 3.9266 \\
\hline 50 & 84 & 45 & 0.7 & Feasible & ulation & Adaptive & asible & Scattered \\
\hline
\end{tabular}


Genetic Algorithm Parameters Optimization For Electrochemical Machining Using Response Surface

Methodology

\begin{tabular}{|c|c|c|c|c|c|c|c|}
\hline 60 & 85 & 45 & 1.0 & Uniform Uniform & Single point & 0.7145 & 3.889 \\
\hline 24 & 86 & 45 & 0.7 & Feasible population & Uniform & Scattered & 0.709 \\
\hline \multicolumn{8}{|l|}{3.9078} \\
\hline 47 & 87 & 45 & 1.0 & Feasible population & & Scattered \\
\hline 0.7038 & 3.93 & & & & \multicolumn{2}{|c|}{ Adaptive feasible } & \\
\hline 6 & 88 & 80 & 0.7 & Uniform Uniform & Scattered & 0.7271 & 3.8661 \\
\hline 66 & 89 & 20 & 0.4 & Feasible population & Uniform & Single point & 0.6978 \\
\hline \multicolumn{8}{|l|}{3.9388} \\
\hline 73 & 90 & 45 & 1.0 & Feasible population & Uniform & Single point & 0.725 \\
\hline \multicolumn{8}{|l|}{3.884} \\
\hline 48 & 91 & 45 & 0.7 & Feasible population & \multicolumn{2}{|c|}{ Adaptive feasible } & Scattered \\
\hline 0.746 & 3.837 & & & & & & \\
\hline 51 & 92 & 45 & 0.7 & Feasible population & \multicolumn{2}{|c|}{ Adaptive feasible } & Scattered \\
\hline 0.746 & 3.8492 & & & & & & \\
\hline 147 & 93 & 70 & 0.9 & Feasible population & \multicolumn{2}{|c|}{ Adaptive feasible } & Two point \\
\hline 0.746 & 3.838 & & & & & & \\
\hline 115 & 94 & 45 & 0.7 & Uniform Uniform & Two point & 0.737 & 3.8654 \\
\hline 101 & 95 & 45 & 0.7 & Feasible population & \multicolumn{2}{|c|}{ Adaptive feasible } & Single point \\
\hline 0.746 & 3.837 & & & & & & \\
\hline 76 & 96 & 45 & 0.7 & \multirow{2}{*}{ Feasible population } & Uniform & Single point & 0.734 \\
\hline 3.914 & & & & & & & \\
\hline 118 & 97 & 20 & 0.4 & Feasible population & Uniform & Two point & 0.729 \\
\hline 3.904 & & & & & & & \\
\hline 142 & 98 & 45 & 0.7 & Uniform & sible & Two point & 0.7474 \\
\hline 3.838 & & & & & & & \\
\hline 46 & 99 & 45 & 0.3 & Feasible population & Adaptive fea & sible & Scattered \\
\hline 0.7455 & 3.838 & & & & & & \\
\hline 27 & 100 & 20 & 0.4 & Uniform & sible & Scattered & 0.7438 \\
\hline 3.92 & & & & & & & \\
\hline 153 & 101 & 45 & 0.7 & Feasible population & Adaptive fea & sible & Two point \\
\hline 0.746 & 3.831 & & & & & & \\
\hline 10 & 102 & 45 & 0.7 & Uniform & Scattered & 0.6966 & 3.8746 \\
\hline 8 & 103 & 45 & 1.0 & Uniform & Scattered & 0.7058 & 3.8858 \\
\hline 81 & 104 & 20 & 0.9 & Uniform & sible & Single point & 0.7414 \\
\hline 3.8532 & & & & & & & \\
\hline 67 & 105 & 70 & 0.4 & Feasible population & Uniform & Single point & 0.73942 \\
\hline 3.8574 & & & & & & & \\
\hline 57 & 106 & 10 & 0.7 & Uniform Uniform & Single point & 0.6708 & 3.9548 \\
\hline 121 & 107 & 70 & 0.9 & Feasible population & Uniform & Two point & 0.7428 \\
\hline 3.8512 & & & & & & & \\
\hline 28 & 108 & 70 & 0.4 & Uniform & sible & Scattered & 0.748 \\
\hline 3.842 & & & & & & & \\
\hline 74 & 109 & 45 & 0.7 & Feasible population & Uniform & Single point & 0.7312 \\
\hline 3.8808 & & & & & & & \\
\hline 29 & 110 & 20 & 0.9 & Uniform & sible & Scattered & 0.7358 \\
\hline 3.8582 & & & & & & & \\
\hline 33 & 111 & 45 & 0.3 & Uniform & sible & Scattered & 0.746 \\
\hline 3.838 & & & & & & & \\
\hline 108 & 112 & 70 & 0.9 & Uniform Uniform & Two point & 0.7234 & 3.8918 \\
\hline 103 & 113 & 45 & 0.7 & Feasible population & Adaptive fea & sible & Single point \\
\hline 0.744 & 3.831 & & & & & & \\
\hline 114 & 114 & 45 & 0.7 & Uniform Uniform & Two point & 0.748 & 3.868 \\
\hline 26 & 115 & 45 & 0.7 & Feasible population & Uniform & Scattered & 0.7312 \\
\hline 3.8854 & & & & & & & \\
\hline 109 & 116 & 10 & 0.7 & Uniform Uniform & Two point & 0.6886 & 4.0832 \\
\hline 126 & 117 & 45 & 0.7 & Feasible population & Uniform & Two point & 0.7334 \\
\hline 3.8668 & & & & & & & \\
\hline 105 & 118 & 20 & 0.4 & Uniform & Two point & 0.7386 & 3.8882 \\
\hline
\end{tabular}




\begin{tabular}{|c|c|c|c|c|c|c|c|c|}
\hline $\begin{array}{l}72 \\
3.8958\end{array}$ & 119 & 45 & 0.3 & \multicolumn{2}{|c|}{ Feasible population } & Uniform & Single point & 0.7176 \\
\hline 144 & 120 & 20 & 0.4 & \multirow{2}{*}{\multicolumn{2}{|c|}{ Feasible population }} & \multirow{2}{*}{\multicolumn{2}{|c|}{ Adaptive feasible }} & Two point \\
\hline 0.7496 & 3.8406 & & & & & & & \\
\hline $\begin{array}{l}90 \\
3.8378\end{array}$ & 121 & 45 & 0.7 & Uniform & \multicolumn{2}{|c|}{ Adaptive feasible } & Single point & 0.7452 \\
\hline $\begin{array}{l}36 \\
3.8392\end{array}$ & 122 & 45 & 0.7 & Uniform & \multicolumn{2}{|c|}{ Adaptive feasible } & Scattered & 0.746 \\
\hline 110 & 123 & 80 & 0.7 & Uniform & Uniform & Two point & 0.7363 & 3.8726 \\
\hline $\begin{array}{l}31 \\
3.96\end{array}$ & 124 & 10 & 0.7 & Uniform & \multicolumn{2}{|c|}{ Adaptive feasible } & Scattered & 0.715 \\
\hline 12 & 125 & 45 & 0.7 & Uniform & Uniform & Scattered & 0.7242 & 3.8571 \\
\hline $\begin{array}{l}32 \\
3.841\end{array}$ & 126 & 80 & 0.7 & Uniform & \multicolumn{2}{|c|}{ Adaptive feasible } & Scattered & 0.7452 \\
\hline $\begin{array}{l}137 \\
3.8388\end{array}$ & 127 & 45 & 0.3 & Uniform & \multicolumn{2}{|c|}{ Adaptive feasible } & Two point & 0.7458 \\
\hline $\begin{array}{l}49 \\
0.746\end{array}$ & $\begin{array}{l}128 \\
3.837\end{array}$ & 45 & 0.7 & \multicolumn{2}{|c|}{ Feasible population } & \multicolumn{2}{|c|}{ Adaptive feasible } & Scattered \\
\hline $\begin{array}{l}148 \\
0.6882\end{array}$ & $\begin{array}{l}129 \\
3.9378\end{array}$ & 10 & 0.7 & \multicolumn{2}{|c|}{ Feasible population } & \multicolumn{2}{|c|}{ Adaptive feasible } & Two point \\
\hline $\begin{array}{l}38 \\
3.837\end{array}$ & 130 & 45 & 0.7 & Uniform & \multicolumn{2}{|c|}{ Adaptive feasible } & Scattered & 0.746 \\
\hline $\begin{array}{l}21 \\
3.893\end{array}$ & 131 & 45 & 1.0 & \multicolumn{2}{|c|}{ Feasible population } & Uniform & Scattered & 0.7142 \\
\hline $\begin{array}{l}39 \\
3.8382\end{array}$ & 132 & 45 & 0.7 & Uniform & \multicolumn{2}{|c|}{ Adaptive feasible } & Scattered & 0.746 \\
\hline 11 & 133 & 45 & 0.7 & Uniform & Uniform & Scattered & 0.726 & 3.8842 \\
\hline $\begin{array}{l}44 \\
0.7296\end{array}$ & $\begin{array}{l}134 \\
3.9048\end{array}$ & 10 & 0.7 & \multicolumn{2}{|c|}{ Feasible population } & \multicolumn{2}{|c|}{ Adaptive feasible } & Scattered \\
\hline $\begin{array}{l}80 \\
3.837\end{array}$ & 135 & 70 & 0.4 & Uniform & \multicolumn{2}{|c|}{ Adaptive feasible } & Single point & 0.746 \\
\hline $\begin{array}{l}152 \\
0.7496\end{array}$ & $\begin{array}{l}136 \\
3.8478\end{array}$ & 45 & 0.7 & \multicolumn{2}{|c|}{ Feasible population } & \multicolumn{2}{|c|}{ Adaptive feasible } & Two point \\
\hline $\begin{array}{l}99 \\
0.746\end{array}$ & $\begin{array}{l}137 \\
3.838\end{array}$ & 45 & 1.0 & \multicolumn{2}{|c|}{ Feasible population } & \multicolumn{2}{|c|}{ Adaptive feasible } & Single point \\
\hline $\begin{array}{l}34 \\
3.8182\end{array}$ & 138 & 45 & 1.0 & Uniform & \multicolumn{2}{|c|}{ Adaptive feasible } & Scattered & 0.7138 \\
\hline $\begin{array}{l}136 \\
3.8403\end{array}$ & 139 & 80 & 0.7 & Uniform & \multicolumn{2}{|c|}{ Adaptive feasible } & Two point & 0.7477 \\
\hline $\begin{array}{l}146 \\
0.7354\end{array}$ & $\begin{array}{l}140 \\
3.8702\end{array}$ & 20 & 0.9 & \multicolumn{2}{|c|}{ Feasible population } & \multicolumn{2}{|c|}{ Adaptive feasible } & Two point \\
\hline $\begin{array}{l}43 \\
0.7426\end{array}$ & $\begin{array}{l}141 \\
3.8434\end{array}$ & 70 & 0.9 & \multicolumn{2}{|c|}{ Feasible population } & \multicolumn{2}{|c|}{ Adaptive feasible } & Scattered \\
\hline 54 & 142 & 70 & 0.4 & Uniform & Uniform & Single point & 0.7278 & 3.8792 \\
\hline $\begin{array}{l}93 \\
0.746\end{array}$ & $\begin{array}{l}143 \\
3.838\end{array}$ & 70 & 0.4 & Feasible $\mathrm{p}$ & ulation & Adaptive fe & sible & Single point \\
\hline $\begin{array}{l}123 \\
3.8612\end{array}$ & 144 & 80 & 0.7 & Feasible $p$ & ulation & Uniform & Two point & 0.7398 \\
\hline $\begin{array}{l}84 \\
3.8402\end{array}$ & 145 & 80 & 0.7 & Uniform & Adaptive & asible & Single point & 0.7447 \\
\hline 56 & 146 & 70 & 0.9 & Uniform & Uniform & Single point & 0.7294 & 3.8898 \\
\hline $\begin{array}{l}133 \\
3.8548\end{array}$ & 147 & 20 & 0.9 & Uniform & Adaptive & asible & Two point & 0.7466 \\
\hline $\begin{array}{l}15 \\
3.856\end{array}$ & 148 & 70 & 0.4 & Feasible $p$ & ulation & Uniform & Scattered & 0.738 \\
\hline $\begin{array}{l}45 \\
0.7451\end{array}$ & $\begin{array}{l}149 \\
3.8404\end{array}$ & 80 & 0.7 & Feasible $\mathrm{p}$ & ulation & Adaptive fe & sible & Scattered \\
\hline 3 & 150 & 20 & 0.9 & Uniform & Uniform & Scattered & 0.69 & 3.91 \\
\hline 58 & 151 & 80 & 0.7 & Uniform & Uniform & Single point & 0.729 & 3.8718 \\
\hline
\end{tabular}


Genetic Algorithm Parameters Optimization For Electrochemical Machining Using Response Surface

Methodology

\begin{tabular}{|c|c|c|c|c|c|c|c|c|}
\hline 79 & 152 & 20 & 0.4 & Uniform & \multicolumn{2}{|c|}{ Adaptive feasible } & Single point & 0.7422 \\
\hline $\begin{array}{l}129 \\
38696\end{array}$ & 153 & 45 & 0.7 & \multicolumn{2}{|c|}{ Feasible population } & Uniform & Two point & 0.7314 \\
\hline $\begin{array}{l}88 \\
3.837\end{array}$ & 154 & 45 & 0.7 & Uniform & \multicolumn{2}{|c|}{ Adaptive feasible } & Single point & 0.746 \\
\hline $\begin{array}{l}119 \\
3.872\end{array}$ & 155 & 70 & 0.4 & \multicolumn{2}{|c|}{ Feasible population } & Uniform & Two point & 0.731 \\
\hline & 156 & 45 & 0.7 & Uniform & Uniforı & Single po & 0.734 & 3.94 \\
\hline
\end{tabular}

\section{RESULT AND DISCUSSIONS}

In this work optimization of parameters of multi-objective genetic algorithm for maximizing the MRR and minimize the SR in ECM machine like population size, crossover, mutation, creation function etc. 5 level Central Composite Design (CCD) of Response Surface Methodology (RSM) of Design Expert software have been considered for optimizing the different multiobjective GA constraints for maximizing the Material Removal Rate(MRR) and minimizing Surface roughness of ECM machine. Table 2 shows the parameters of GA with their range and levels ,which are taken into consideration in this work. 156 experiments have been provided by the design expert through the combination of various parameters which are to be performed for the optimization. Each combinational set is run five times and the average of these taken as the final result. Hence total $156 * 5=780$ outcomes were used for the optimization of GA constraints. Design summary is shown below in table 3. In which 2 factors (A and $\mathrm{B}$ ) are numeric and other 3 factors (C, D and $\mathrm{E})$ are definite.

Table 2: Genetic Algorithm Parameters With Range And Levels

\begin{tabular}{|l|l|l|}
\hline S no. & Parameters & Range \\
\hline 1 & Population size (A) & $20-70(5$ Levels) \\
\hline 2 & Crossover fraction(B) & $0.4-0.9(5$ Levels) \\
\hline 3 & Creation function(C) & Level 1(Uniform),Level 2(Feasible population) \\
\hline 4 & Mutation function(D) & Level 1(Uniform),Level 2(Adaptive feasible) \\
\hline 5 & Crossover function(E) & Level 1(Scattered), Level 2(Single point), Level 3(Double point) \\
\hline
\end{tabular}

Table 3: Design Review

\begin{tabular}{|c|c|c|c|c|c|c|c|c|c|}
\hline \multirow{3}{*}{\multicolumn{2}{|c|}{$\begin{array}{l}\text { Type of study } \\
\text { Initial Design } \\
\text { Design Model }\end{array}$}} & \multirow{3}{*}{\multicolumn{2}{|c|}{$\begin{array}{l}\text { Response Surface } \\
\text { Central Composite } \\
\text { Quadratic }\end{array}$}} & & \multicolumn{2}{|c|}{ Experiments } & \multirow[t]{3}{*}{156} & & \\
\hline & & & & & \multirow{2}{*}{\multicolumn{2}{|c|}{ Blocks No Blocks }} & & & \\
\hline & & & & & & & & & \\
\hline Response & Name & Units & Obs & Minimum & \multicolumn{2}{|l|}{ Maximum } & \multicolumn{3}{|l|}{ Model } \\
\hline Y1 & MRR & $\mathrm{g}$ & 156 & 0.67 & \multicolumn{2}{|l|}{0.75} & \multicolumn{3}{|l|}{ Quadratic } \\
\hline Y2 & SR & micrometer & 156 & 3.80 & \multicolumn{2}{|l|}{4.10} & \multicolumn{3}{|l|}{ Quadratic } \\
\hline Factor & Name & Units & Type & Low Actual & \multicolumn{2}{|c|}{ High Actual Low Coded } & \multicolumn{3}{|c|}{ High Coded } \\
\hline A & \multicolumn{2}{|c|}{ Population size } & & Numeric & 20.00 & 70.00 & -1.000 & 1.000 & \\
\hline B & \multirow{2}{*}{\multicolumn{2}{|c|}{$\begin{array}{l}\text { Crossover Fraction } \\
\text { Creation function }\end{array}$}} & Numeric & 0.40 & 0.90 & -1.000 & 1.000 & & \\
\hline $\mathrm{C}$ & & & Categorical & Uniform & \multicolumn{2}{|c|}{ Feasible population } & Levels: & 2 & \\
\hline D & \multicolumn{2}{|c|}{ Mutation function } & Categorical & Uniform & \multicolumn{2}{|c|}{ Adaptive feasible } & & Levels: & 2 \\
\hline $\mathrm{E}$ & \multicolumn{2}{|c|}{ Crossover function } & Categorical & Scattered & \multicolumn{2}{|c|}{ Two point } & Levels: & 3 & \\
\hline
\end{tabular}

A. ANOVA for the Response Surface Quadratic Model

ANOVA test have been conducted for the optmisation of the MRR and SR as shown in table 4 and 5.The model used for optimizing process parameters of GA is quadratic in nature. The F-test and probability test have been performed for checking the significance. The F-ratio is the fraction between groups means square values to within group mean square values. The Pvalues have been compared with each coefficient to check its significance. If P- value is less than 0.05 for a planned model, then it is significant at the 5\% level of significance. It is to be noted that Lack of fit is not significant in both cases.

TABLE 4: ANOVA for Response Surface Quadratic Model of MRR 


\begin{tabular}{|c|c|c|c|c|c|c|}
\hline \multirow{2}{*}{ F } & \multirow[t]{2}{*}{$\begin{array}{l}\text { Sum of } \\
\text { Squares }\end{array}$} & \multicolumn{2}{|c|}{$\begin{array}{l}\text { Degree of } \\
\text { freedom }\end{array}$} & \multirow[t]{2}{*}{$\begin{array}{l}\text { Mean } \\
\text { Square }\end{array}$} & \multirow[t]{2}{*}{$\begin{array}{c}\text { F } \\
\text { Value }\end{array}$} & \multirow[t]{2}{*}{ Prob > } \\
\hline & & & & & & \\
\hline \multirow{2}{*}{\multicolumn{2}{|c|}{$\begin{array}{l}\text { Model } \\
\text { A } 9.069 \times 10^{-0035}\end{array}$}} & 22 & $1.604 \times 10^{-003}$ & 13.69 & $<0.0001$ & \multirow[t]{4}{*}{ significant } \\
\hline & & 1 & $9.069 \times 10^{-003}$ & 77.38 & $<0.0001$ & \\
\hline \multicolumn{2}{|c|}{$\mathrm{B} 1.795 \times 10^{-003}$} & 1 & $1.795 \times 10^{-003}$ & 15.32 & 0.0001 & \\
\hline \multicolumn{2}{|c|}{ C9.901 x10 000} & 1 & $9.901 \times 10^{-008}$ & $8.448 \times 10^{-004}$ & 0.9769 & \\
\hline & 0.014 & & 1 & 0.014 & 121.89 & \multirow[t]{2}{*}{$<0.0001$} \\
\hline \multicolumn{2}{|c|}{ E1.395 x10-003 } & 2 & $6.974 \times 10^{-004}$ & 5.95 & 0.0033 & \\
\hline \multicolumn{2}{|c|}{$\mathrm{A}^{2} 4.714 \times 10^{-003}$} & 1 & $4.714 \times 10^{-003}$ & 40.23 & $<0.0001$ & \\
\hline \multicolumn{2}{|c|}{$\mathrm{B}^{2} 3.333 \times 10^{-004}$} & 1 & $3.333 \times 10^{-004}$ & 2.84 & 0.0941 & \\
\hline \multicolumn{2}{|c|}{$\mathrm{AB} 4.951 \times 10^{-005}$} & 1 & $4.951 \times 10^{-005}$ & 0.42 & 0.5168 & \\
\hline \multicolumn{2}{|c|}{ AC4.175 x10-004 } & 1 & $4.175 \times 10^{-004}$ & 3.56 & 0.0613 & \\
\hline \multicolumn{2}{|c|}{ AD1.382 $\times 10^{-003}$} & 1 & $1.382 \times 10^{-003}$ & 11.80 & 0.0008 & \\
\hline \multicolumn{2}{|c|}{$\mathrm{AE} 1.043 \times 10^{-005}$} & 2 & $5.216 \times 10^{-006}$ & 0.045 & 0.9565 & \\
\hline \multicolumn{2}{|c|}{ BC4.436 x $10^{-007}$} & 1 & $4.436 \times 10^{-007}$ & $3.785 \times 10^{-003}$ & 0.9510 & \\
\hline \multicolumn{2}{|c|}{ BD2.387 x10 $0^{-007}$} & 1 & $2.387 \times 10^{-007}$ & $2.036 \times 10^{-003}$ & 0.9641 & \\
\hline \multicolumn{2}{|c|}{ BE7.797 x $10^{-004}$} & 2 & $3.898 \times 10^{-004}$ & 3.33 & 0.0389 & \\
\hline \multicolumn{2}{|c|}{ CD7.387 x10 $0^{-005}$} & 1 & $7.387 \times 10^{-005}$ & 0.63 & 0.4286 & \\
\hline \multicolumn{2}{|c|}{ CE2.498 $\times 10^{-004}$} & 2 & $1.249 \times 10^{-004}$ & 1.07 & 0.3475 & \\
\hline \multicolumn{2}{|c|}{ DE9.799 $\times 10^{-004}$} & 2 & $4.899 \times 10^{-004}$ & 4.18 & 0.0173 & \\
\hline Residual & 0.016 & 133 & $1.172 \times 10^{-004}$ & & & \\
\hline Lack of Fit & 0.011 & 85 & $1.300 \times 10^{-004}$ & 1.38 & 0.1146 & not significant \\
\hline \multicolumn{2}{|c|}{ Pure Error $4.534 \times 10^{-003}$} & 48 & $9.446 \times 10^{-005}$ & & & \\
\hline Cor Total & 0.051 & 155 & & & & \\
\hline
\end{tabular}

F-value of 13.69 of this model mean that the model is significant and there is only a $0.01 \%$ chance that a "Model F-Value" this large could occur due to noise.Values of "Prob > F" is less than 0.0500 specify model terms are considerablely. A, B, D, $\mathrm{E}, \mathrm{A}^{2}, \mathrm{AD}, \mathrm{BE}, \mathrm{DE}$ are significant model expressions.Values greater than 0.1000 specify that the model provisions are not significant.

Table 5: Anova For Response Surface Quadratic Model Of Sr

\begin{tabular}{|c|c|c|c|c|c|}
\hline $\begin{array}{r}\text { Source } \\
\text { Prob }>\text { F }\end{array}$ & $\begin{array}{l}\text { Sum of } \\
\text { Squares }\end{array}$ & $\begin{array}{c}\text { Degree of } \\
\text { freedom }\end{array}$ & & $\begin{array}{l}\text { Mean } \\
\text { Square }\end{array}$ & $\begin{array}{c}\mathbf{F} \\
\text { Value }\end{array}$ \\
\hline Model & 0.26 & 22 & 0.012 & 10.82 & $<0.0001$ \\
\hline A & 0.12 & 1 & 0.12 & 107.74 & $<0.0001$ \\
\hline $\mathrm{B}$ & $1.432 \times 10^{-004}$ & 1 & $1.432 \times 10^{-004}$ & 0.13 & 0.7197 \\
\hline $\mathrm{C}$ & $6.972 \times 10^{-004}$ & 1 & $6.972 \times 10^{-004}$ & 0.63 & 0.4290 \\
\hline $\mathrm{D}$ & 0.073 & 1 & 0.073 & 65.57 & $<0.0001$ \\
\hline $\mathrm{E}$ & $2.071 \times 10^{-003}$ & 2 & $1.036 \times 10^{-003}$ & 0.93 & 0.3952 \\
\hline$A^{2}$ & 0.047 & 1 & 0.047 & 42.60 & $<0.0001$ \\
\hline $\mathrm{B}^{2}$ & $1.741 \times 10^{-005}$ & 1 & $1.741 \times 10^{-005}$ & 0.016 & 0.9004 \\
\hline $\mathrm{AB}$ & $2.715 \times 10^{-003}$ & 1 & $2.715 \times 10^{-003}$ & 2.45 & 0.1198 \\
\hline $\mathrm{AC}$ & $1.723 \times 10^{-004}$ & 1 & $1.723 \times 10^{-004}$ & 0.16 & 0.6939 \\
\hline $\mathrm{AD}$ & $6.082 \times 10^{-003}$ & 1 & $6.082 \times 10^{-003}$ & 5.49 & 0.0206 \\
\hline $\mathrm{AE}$ & $1.494 \times 10^{-003}$ & 2 & $7.470 \times 10^{-004}$ & 0.67 & 0.5112 \\
\hline $\mathrm{BC}$ & $2.536 \times 10^{-004}$ & 1 & $2.536 \times 10^{-004}$ & 0.23 & 0.6331 \\
\hline $\mathrm{BD}$ & $1.148 \times 10^{-004}$ & 1 & $1.148 \times 10^{-004}$ & 0.10 & 0.7480 \\
\hline
\end{tabular}


Genetic Algorithm Parameters Optimization For Electrochemical Machining Using Response Surface

Methodology

$\begin{array}{lccccc}\text { BE } & 1.766 \times 10^{-003} & 2 & 8.832 \times 10^{-004} & 0.80 & 0.4527 \\ \mathrm{CD} & 8.252 \times 10^{-004} & 1 & 8.252 \times 10^{-004} & 0.75 & 0.3896 \\ \mathrm{CE} & 6.124 \times 10^{-003} & 2 & 3.062 \times 10^{-003} & 2.76 & 0.0666 \\ \mathrm{DE} & 1.556 \times 10^{-003} & 2 & 7.782 \times 10^{-004} & 0.70 & 0.4971 \\ \text { Residual } & 0.15 & & 133 & 1.108 \times 10^{-003} & 0.4467 \\ \text { Lack of Fit } & 0.096 & 85 & 1.124 \times 10^{-003} & 1.04 & \\ \text { significant } & & & & & \\ \text { Pure Error } & 0.052 & 48 & 1.079 \times 10^{-003} & & \\ \text { Cor Total } & 0.41 & & 155 & \end{array}$

The Model F-value of 10.82 implies the model is significant. A, D, $\mathrm{A}^{2}$, AD are significant model terms.

\section{B. Optimum GA Parameters Predicted by RSM}

Optimum parameters have been obtained using Central Composite Design (CCD) of Response Surface Methodology (RSM) of Design Expert software. Total of 156 experiments have been carried out for the optmisation of the MRR and SR simultaneously for ECM machine. Criteria for optimization and Optimum parameter selection for MRR and SR have been shown in table 6 and table 7.Through the various experiments the optimum parameters have been found to be population size: 59, Crossover Fraction: 0.4,creation function: Feasible population, mutation: Adaptive feasible, creation function: Two point and selection: tournament.

Table 6: Criteria For Optimization

\begin{tabular}{lllll}
\hline Name & Goal & Lower & Upper & Importance \\
\hline- & & & & \\
Population size $\quad$ is in range & 20 & 70 & 3 & 3 \\
Crossover Fraction is in range & 0.4 & 0.9 & 3 \\
Creation function & is in range & Uniform & Feasible population & \\
Mutation function & is in range & Uniform & Adaptive feasible & 3 \\
Crossover function is in range & Scattered & Two point & 3 \\
MRR $\quad$ maximize & 0.6708 & 0.7542 & 3 \\
SR & minimize & 3.7984 & 4.1 & \\
\hline
\end{tabular}

Table 7: Optimum Parameter Selection For MRR And SR

\begin{tabular}{|c|c|c|c|c|c|c|c|c|}
\hline \multirow{2}{*}{ NO. } & \multirow{2}{*}{$\begin{array}{c}\text { Population } \\
\text { size }\end{array}$} & \multirow{2}{*}{$\begin{array}{l}\text { Crossover } \\
\text { Fraction }\end{array}$} & \multirow{2}{*}{$\begin{array}{l}\text { Creation } \\
\text { function }\end{array}$} & \multirow{2}{*}{$\begin{array}{c}\text { Mutation } \\
\text { function }\end{array}$} & \multirow[t]{2}{*}{ MRR } & \multirow{2}{*}{\multicolumn{2}{|c|}{ SR }} & \multirow[t]{2}{*}{ Desirability } \\
\hline & & & & & & & & \\
\hline 1 & 59 & $0.4 \quad \mathrm{~F}$ & Feasible p. & Adaptive feasible Two point & 0.749454 & 3.81884 & 0.938 & Selected \\
\hline 2 & 66 & $0.4 \quad F$ & Feasible p. & Adaptive feasible Scattered & 0.7542 & 3.83659 & 0.935 & \\
\hline 3 & 58 & 0.4Uniforr & rm Adapt & tive feasibleScattered 0.750998 & 3.82765 & 0.932 & & \\
\hline 4 & 57 & 0.4 Uniforr & Adapt & tive feasibleScattered0.751071 & 3.82792 & 0.932 & & \\
\hline 5 & 57 & 0.4 Uniforr & Adapt & tive feasibleScattered0.751121 & 3.82812 & 0.932 & & \\
\hline 6 & 55 & 0.4Uniforr & $\mathrm{rm} \quad$ Adapt & tive feasible Two point 0.75164 & 3.83767 & 0.918 & & \\
\hline 7 & 59 & $0.5 \quad \mathrm{~F}$ & Feasible p. & Adaptive feasible Single point & 0.746955 & 3.82579 & 0.911 & \\
\hline 8 & 54 & 0.6Uniforr & $\mathrm{rm} \quad$ Adapt & tive feasible Single point 0.7484 & 4423.83572 & 0.903 & & \\
\hline 9 & 54 & 0.6Uniforr & $\mathrm{rm} \quad$ Adapt & tive feasible Single point 0.7484 & 4383.83571 & 0.903 & & \\
\hline 10 & 70 & $0.4 \quad F$ & Feasible p.Un & Iniform $\quad$ Two point0.7417 & 993.8443 & 0.850 & & \\
\hline
\end{tabular}

\section{CONCLUSION}

In this research paper, parametric optmisation for Genetic Algorithm for the maximization of the MRR and minimizationof the SR have been done. The efficiency and effectiveness of GA is primarily depends on its various parameters like population size, crossover fraction, creation function, mutation, crossover function etc. For the optimization of these GA parameters a 5 level numeric factor Central Composite Design (CCD) of Response surface methodology (RSM). Total 156 experiments are performed by varying its various GA parameters in MATLAB environment for the optimal result. The optimum values for maximizing the MRR and minimizing the SR simultaneously are Population size: 59, Crossover Fraction: 0.4, Creation function: Feasible population, Mutation: Adaptive feasible, Creation function: Two point. 


\section{REFERENCES}

[1] https://en.wikipedia.org/wiki/Electrochemical_machining.

[2] B. Bhattacharyya, M. Malapati , J. Munda, 2005. "Experimental study on electrochemical micromachining", Journal of Materials Processing Technology 169 (2005) 485-49

[3] N. K. Jain \& V. K. Jain, 2007. "Optimization of electro chemical machining process parameters using Genetic Algorithms", Machining Science and Technology ISSN: 1091-0344 (Print) 1532-2483

[4] D. Datta and A.k. Das, 2010."Tuning process parameters of Electrochemical machining using a Multi-objective Genetic algorithm", Springer-Verlag Berlin Heidelberg 2010 LNCS 6457, pp. 485-493,

[5] C. Senthilkumar, G. Ganesan, R.K. , 2012."Optimization of ECM process parameters using NSGA-II", Journal of Minerals and Materials Characterization and Engineering, 2012, 11, 931-937

[6] B.R. Acharya,C.P. Mohanty, S.S Mahapatra ,2013."Multi-objective optimization of electrochemical machining of hardened steel using NSGA-II", Procedia Engineering 51 (2013) $554-560$

[7] A Giribabu, S. R. Rao and G Padmanbhan, 2014."Optimization of machining parameters in ECM of Al/B4C Composites using Genetic algorithms", IJMERR, July 2014

[8] M. Sankar, A. Gnanavelbabu and R.Baskaran,2014."Optimization of surface roughness in Electro chemical machining,"Trans Tech Publications, Switzerland Applied Mechanics and Materials Vol. 606 pp 193-197

[9] V. Sathiyamoorthy, T. Sekar, and N. Elango, 2015."Optimization of process parameters in ECM of die tool steel using nanofluid by Multi-objective Genetic algorithm", Hindawi Publishing Corporation, The Scientific World Journal Volume 2015, Article ID 895696

[10] Abhishek, A. Mandal, Kaushik, 2015."Multi-objective optimization of Electro-chemical machining by non-dominated sorting Genetic algorithm", Materials Today: Proceedings 2 ( 2015 ) $2569-2575$

[11] http://novelmech.com/2016/05/electrochemical-machining.html.

[12] http://novelmech.com/2016/05/electrochemical-machining.html. 